\title{
Novel therapeutic strategies and perspectives for metastatic pancreatic cancer: vaccine therapy is more than just a theory
}

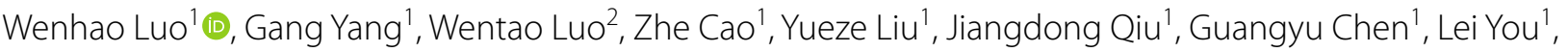 \\ Fangyu Zhao ${ }^{1}$, Lianfang Zheng ${ }^{3}$ and Taiping Zhang ${ }^{1,4^{*}}$
}

\begin{abstract}
Pancreatic cancer is an aggressive and malignant tumor with an exceedingly high mortality rate. The quality of life and survival rates of pancreatic cancer patients with metastasis are poor compared with those without metastasis. Thus far, no effective treatment strategy has been established for metastatic pancreatic cancer patients. Therefore, an appropriate therapeutic method based on the elimination of metastatic pancreatic cancer is critical to improve patient outcome. Tumor-targeted vaccines have been widely discussed in recent studies and enabled important breakthroughs in the treatment of pancreatic cancer by preventing the escape of tumor cells from immune surveillance and activating the immune system to eliminate cancer cells. T cells can be activated by the stimulation of tumor-targeted vaccines, but to mount an effective immune response, both immune checkpoint inhibitors and positive costimulatory molecules are required. In this review, we discuss potential tumor-targeted vaccines that can target pancreatic cancer, elaborate the probably appropriate combination of vaccines therapy and evaluate the underlying benefits as well as obstacles in the current therapy for metastatic pancreatic cancer.
\end{abstract}

Keywords: Vaccination, Pancreatic cancer, Metastasis, Immune therapy, Novel strategies

\section{Background}

Pancreatic cancer (PC) is an aggressive disease with a poor 5-year survival rate that is mainly attributed to metastasis. PC is often diagnosed at an advanced stage, because the clinical symptoms are not obvious. Chemotherapy is not always successful. Hence, surgery with radical resection is presently the only curative therapy for PC patients. However, less than $20 \%$ of PC patients are eligible for operation because of disease progression and metastases [1]. Additionally, because of difficulties in full

\footnotetext{
*Correspondence: tpingzhang@yahoo.com

1 Department of General Surgery, Peking Union Medical College Hospital, Chinese Academy of Medical Sciences and Peking Union Medical College, No. 1 Shuaifuyuan, Wangfujing Street, Beijing 100730, China

Full list of author information is available at the end of the article
}

elimination of PC with surgical resection or chemo-radiotherapy, metastatic PC is currently an unmanageable disease. Therefore, developing novel therapies for metastatic PC is critical.

Immune therapies are classified into active immune such as vaccines therapy and passive immune (or adaptive immune) therapy such as antibodies. Active immune therapies involves a process whereby vaccines target the tumor antigens to enable the patient to mount an immune response and develop immunologic memory. Vaccine-associated immunotherapy is a new treatment strategy in cancer research. Tumor-associated vaccines can inhibit the migration of cancer cells through strengthened immune surveillance. However, the

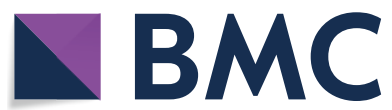

(c) The Author(s) 2020. This article is licensed under a Creative Commons Attribution 4.0 International License, which permits use, sharing, adaptation, distribution and reproduction in any medium or format, as long as you give appropriate credit to the original author(s) and the source, provide a link to the Creative Commons licence, and indicate if changes were made. The images or other third party material in this article are included in the article's Creative Commons licence, unless indicated otherwise in a credit line to the material. If material is not included in the article's Creative Commons licence and your intended use is not permitted by statutory regulation or exceeds the permitted use, you will need to obtain permission directly from the copyright holder. To view a copy of this licence, visit http://creativeco mmons.org/licenses/by/4.0/. The Creative Commons Public Domain Dedication waiver (http://creativecommons.org/publicdomain/ zero/1.0/) applies to the data made available in this article, unless otherwise stated in a credit line to the data. 
influence of tumor-targeted vaccines on metastasis in PC remains unclear.

This article reviews newly discovered risk factors that are related to metastatic PC along with recent studies on tumor-associated vaccine therapies with the aim of finding more accurate strategies for vaccine therapies towards metastatic PC (Table 1).

\section{Vaccines, tumor-associated antigens and cancer therapy \\ Vaccines and PC treatment}

Several kinds of cancer vaccines are available, including whole cell vaccines, peptide-based vaccines, dendritic cell (DC) vaccines, DNA vaccines (plasmid vaccines, virus-based vaccines, bacterial vectors as well as yeastbased recombination vaccines) and mRNA vaccines. At present, suppressed and damaged immune system in PC patients are great challenges for cancer vaccines because of the malignancy of cancer, the adverse impacts of chemo- or radio-therapies as well as the advanced stage of PC. However, cancer vaccination involves various strategies to amplify anti-cancer immunity, including the administration of tumor antigens, often with antigen presenting cells (APCs) such as DCs or other immune modulators, or direct modulation of the tumor. Elimination of metastatic PC mainly relies on cytotoxic drugs or cytotoxic immune cells such as CD8+ T cells that kill tumor cells or hinder their proliferation. Nearly all cancer vaccines realize their killing effects by activating tumorspecific CD8+ cytotoxic $\mathrm{T}$ cells based on the delivery of MHC class I restricted peptide epitopes derived from shared antigens expressed on the tumor.

In a recent multicenter Phase II study, the peptide cocktail vaccine OCV-C01 combined with gemcitabine (a current first-line chemotherapy) in PC patients $(\mathrm{n}=30)$ showed a median Disease-free survival (DFS) of 15.8 months, which was an improvement compared with gemcitabine alone (a DFS of 12.0 months) [2]. Hence, therapeutic strategies involving the combination of chemotherapy with vaccines may promote the levels of cancer-specific T-cells in immunogenic cancers with stronger outcomes.

\section{Tumor-associated antigens and PC therapy}

Recent studies have shown that PC is an immunogenic tumor and researches on antibodies targeting tumor cells have increased [3]. Antibodies can enhance killing effects of immune-related cells by recognizing tumor-associated antigens (TAAs) expressed on tumor cells [4]. For instance, Dinutuximab, an antibody targeting the TAA ganglioside GD2, has been approved by the FDA [5]. Surprisingly, vaccines targeting TAAs have been reported as potential therapeutic interventions [6]. CA 19-9, also known as Sialyl Lewis, is a carbohydrate TAA that is highly expressed on PC cells [7]. Weitzenfeld et al. used CA 19-9-targeted antibodies produced from the serum of CA 19-9/keyhole limpet hemocyanin (KLH) vaccineimmunized patients to successfully protect mice from PC progression. These results suggest that CA19-9-targeted vaccines could potentially be translated to the clinics [8].

Table 1 Preclinical and clinical trials of cancer vaccines targeting metastasis PC

\begin{tabular}{|c|c|c|c|c|c|}
\hline Vaccines names & Vaccine types & Targeted disease & Trials & Function & References \\
\hline OCV-C01 & Peptide vaccine & Pancreatic cancer & Multicenter Phase II study & $\begin{array}{l}\text { Improve the efficacy of } \\
\text { Gemcitabine to PC } \\
\text { metastasis }\end{array}$ & {$[2]$} \\
\hline $\begin{array}{l}\text { Ganglioside GD2 targeted } \\
\text { vaccine }\end{array}$ & DC vaccine/Peptide vaccine & Pancreatic cancer & FDA approved & $\begin{array}{l}\text { Successfully protect from PC } \\
\text { progression }\end{array}$ & {$[5]$} \\
\hline CA 19-9/KLH vaccine & Conjugate vaccine & Pancreatic cancer & Phase I clinical trials & $\begin{array}{l}\text { Successfully protect from PC } \\
\text { progression }\end{array}$ & {$[8]$} \\
\hline MUC1-peptide DC vaccines & DC vaccine/Peptide vaccine & Pancreatic cancer & Phase I pilot trial & $\begin{array}{l}\text { Enhance immunological } \\
\text { response in metastatic PC }\end{array}$ & {$[16]$} \\
\hline Synthetic ras peptides & Peptide vaccine & Pancreatic cancer & Pilot I/II study & $\begin{array}{l}\text { Enhance immunological } \\
\text { response in metastatic PC }\end{array}$ & {$[19]$} \\
\hline SVN-2B vaccines & Peptide vaccine & Pancreatic cancer & Phase I/II clinical trial & $\begin{array}{l}\text { Enhance immunological } \\
\text { response in metastatic PC }\end{array}$ & {$[22]$} \\
\hline Vaccines CRS-207 & Whole cell vaccine & Pancreatic cancer & Pre-clinical & $\begin{array}{l}\text { Enhance immunological } \\
\text { response in metastatic PC }\end{array}$ & {$[30]$} \\
\hline GVAX vaccination & Whole cell vaccine & Pancreatic cancer & Pre-clinical & $\begin{array}{l}\text { Enhance immunological } \\
\text { response in metastatic PC }\end{array}$ & {$[31]$} \\
\hline PAS vaccine & $\begin{array}{l}\text { DNA vaccine/Peptide } \\
\text { vaccine }\end{array}$ & Pancreatic cancer & Pre-clinical & $\begin{array}{l}\text { Enhance immunological } \\
\text { response in metastatic PC }\end{array}$ & {$[45]$} \\
\hline
\end{tabular}


Currently, CA19-9-targeted antibodies are in phase I clinical trials (NCT02672917).

Notably, TAAs may cause serious autoimmune toxicities, as TAAs are expressed in both normal cells and tumor cells [9]. Therefore, antibodies and effector immune cells induced by TAA-targeting vaccines could attack both cancer cells and normal tissues, resulting in increased toxicity. Above all, the identification of a specific antigen that specifically expressed on tumor cells in metastatic PC patients is a novel strategy to precisely target PC cells with reduced toxicity.

\section{Tumor-specific antigen and PC therapy}

In addition to TAAs, cancer vaccines can target other kinds of antigens such as cancer germline antigens, virusassociated antigens, and tumor-specific antigens (TSAs, neoantigens). Tumor-specific antigens are specifically expressed on cancer cells with a low risk of self-tolerance and autoimmunity, which can be successfully targeted by personalized vaccines [10]. TSAs are cancer-specific and patient-specific to induce a stronger $\mathrm{T}$ cell response without killing normal tissues. Hence, we speculated that cancer vaccines targeting TSAs can render more immunogenic effects on inhibiting PC metastasis. TSAs can make up for the autoimmune toxicities shortcomings of TAAs. Antigens expressed on PC cells are easily mutated due to their inherent genetic instability. Innumerable non-synonymous mutations have been found in TSAs which makes it become "specific". TSAs are not expressed on normal cells and thus do not induce autoimmunity. Therefore, targeting TSAs may represent a relatively safe and effective strategy for cancer vaccines compared with targeting TAAs.

Mechanically, TSAs combine with MHC molecules of APCs and are presented by APCs from the cancer vaccine to $\mathrm{T}$ cells while the APCs migrating to the lymph nodes. Specific T cells recognize the TSAs to enhance activation of $\mathrm{T}$ cell immunity. This activity attracts more specific $\mathrm{T}$ cells infiltrating into the tumor microenvironment (TME) to comprise the T cell receptor/MHC/TSA complex, eventually activating the immune response and tumor killing effects of CD4+ T cells and CD8+ $\mathrm{T}$ cells. Besides, the destroyed tumor cells can release more TSAs, which not only induce an immune memory but also lead to expansion of $\mathrm{T}$ cell activation. BassaniSternberg et al. designed a phase $1 \mathrm{~b}$ trial to determine the feasibility of novel autologous DCs pulsed with personalized TSA peptides in PC patients and found robust CD4+ T cell responses [11]. Therefore, TSA vaccination can generate a highly specific immune response against $\mathrm{PC}$, indicating that a personalized TSA vaccine can bring increased benefits and enhance the function of specific $\mathrm{T}$ cells compared with existing immunotherapeutics. In short, we can produce vaccines targeting TAAs as well as TSAs to produce tumor-targeted antibodies, which can inhibit tumor metastasis and progression, while more novel TSAs of PC need to be further explored.

\section{DC vaccines targeting PC}

\section{How DC vaccines inhibit PC metastasis}

Vaccination strategies for PC aim to enhance immune infiltration and endogenous antigen presentation to effector $\mathrm{T}$ cells. DC vaccines are a current focus in therapeutic development and can be activated by tumor antigen in treating cancers and as a result promoting cytotoxic T-cell response [12]. DC is a member of the APC family that efficiently present antigens to $\mathrm{CD} 4+$ and $\mathrm{CD} 8+$ $\mathrm{T}$ cells and secrete cytokines such as IL-15, IL-12, IFN- $\gamma$ and TNF, which promotes activation of cytotoxic CD8+ $\mathrm{T}$ cells by transforming the immune response type into a type 1 response [13]. The efficacy of DC vaccination is significantly associated with the amount of DC vaccines migrating to the draining lymph nodes. Large quantities of lymph nodes are present in the abdomen including spleen and peritoneum. Intraperitoneal injection of DC vaccines could enhance the anti-tumor immune response as well as immune memory and inhibit PC metastasis, improving clinical outcomes $[14,15]$. Therefore, DC vaccinations may become a potential strategy to inhibit metastasis of $\mathrm{PC}$, and $\mathrm{DC}$ vaccination via intraperitoneal injection for immunotherapy in metastatic PC patients may bring about stronger therapeutic responses (Fig. 1).

\section{Application of DC vaccines in clinics}

DC-based vaccines for PC immunotherapy have been proven clinically safe and efficient to induce tumor-specific immune responses. DC vaccination is a potential immune therapy for metastatic PC and the clinical outcomes are often hopeful. Some studies have investigated the potential effects of DC vaccination targeting metastatic PC. For example, Rong et al. conducted a phase I pilot trial on the MUC1-peptide DC vaccine in metastatic PC patients and found the vaccine enhanced the immunological response to the tumor antigen MUC1 in metastatic PC patients without significant toxicity [16]. Mehrotra et al. found that vaccination with peptidepulsed DCs combined with the toll-like receptor (TLR)-3 agonist poly-ICLC is a potential therapeutic method for inhibiting metastasis of $\mathrm{PC}$ by generating CD8 $+\mathrm{T}$ cells [17]. Liang et al. examined the cytotoxic $\mathrm{T}$ lymphocyte (CTL) responses induced by DC vaccination for $\mathrm{PC}$ with the longitudinal assessment of therapeutic responses and demonstrated that $\mathrm{DC}$ vaccines can efficiently enhance CTL response and inhibit the migration of PC [15]. 


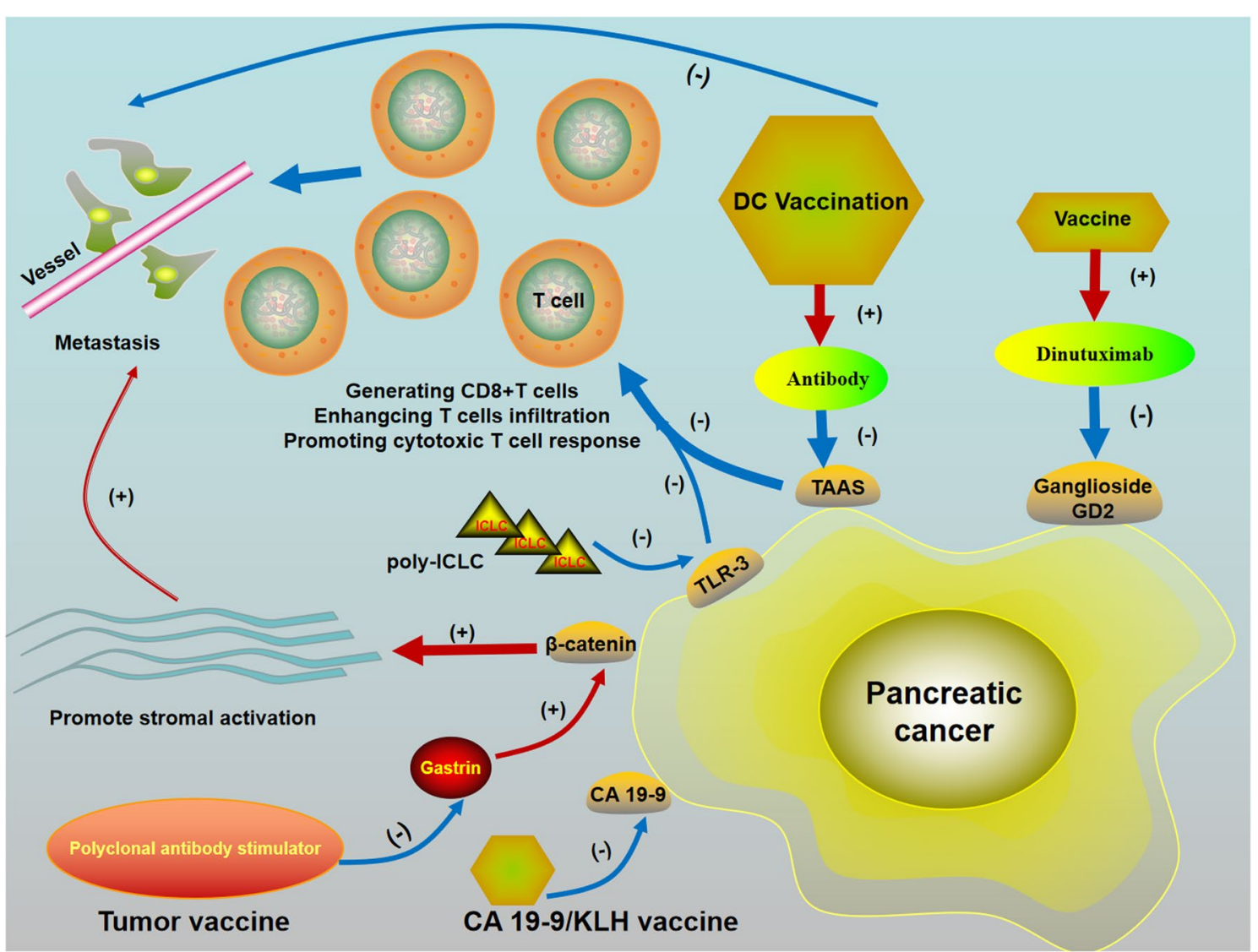

Fig. 1 The killing funtion to metastasis pancreatic cancer (PC) of Cytotoxic T cells are promoted by vaccinations. Vaccine therapies that can improve CD8+ T cell ability which are effective in targeting PC metastasis. 1) vaccination with peptide-pulsed dendritic cell (DC) combined with the toll-like receptor (TLR)-3 agonist poly-ICLC is a potential therapeutic method for inhibiting PC metastasis by generating CD8+T cells; 2) DC vaccines can efficiently enhance cytotoxic T lymphocyte (CTL) response; 3 ) the tumor vaccine polyclonal antibody stimulator, which selectively targets gastrin, induces system to produce specific polyclonal anti-gastrin antibodies and then decrease PC growth and metastases by downregulating dense desmoplastic fibrosis in the TME, inducing T cell activation. 4) CA 19-9-targeted antibodies produced in CA 19-9/keyhole limpet hemocyanin (KLH) vaccine-immunized patients to successfully inhibit PC progression. (+) Promote (-) Inhibit

\section{Peptide-based vaccines targeting PC}

Peptide vaccines are another important type of cancer vaccine that can induce strong immune therapy by improving stimulation of T-cell immunity. Peptide vaccinations induce numerous antigen-specific anticancer immune responses by recognizing and activating $\mathrm{T}$ cells with MHC [18].

Like other vaccines, both specific peptide vaccines and broad peptide vaccines can strengthen effector $\mathrm{T}$ cells to eliminate cancer cells, but each has its own strengths and shortcomings. Specific peptide vaccines target TSAs to generate a strong and targeted anticancer immune response with minimal toxicity. Therefore, a special peptide-based vaccine that specifically targets $P C$ should be pursued in future studies. A pilot I/II study by Gjertsen et al. used synthetic RAS peptides as a cancer vaccine in PC patients and found that a strong immune response could be induced [19].

\section{Peptide-based vaccines and adjuvants}

Research has shown the positive efficacy of numerous immunomodulators combined with peptide vaccines. Various strategies have attempted to enhance peptide immunogenicity. Some reports suggested that stronger efficacy can be achieved by co-treating with cytokines or other immune promoters [20]. The reason is that peptide-based cancer vaccines only incorporate MHCclass I-restricted peptides to activate CD8+ T cells, but the incorporation of $\mathrm{CD} 4+$ helper $\mathrm{T}$ cells activated by other immune agents may also be necessary for PC elimination. For instance, clinical phase I/II trial assessed the efficacy of synthetic mutant RAS peptides in combination with granulocyte-macrophage colonystimulating factor and found that PC patients showed a stronger immune response to the peptide vaccine with prolonged survival compared with standard therapy (median survival 148 days vs. 61 days, respectively; 
$\mathrm{p}=0.0002$ ) [21]. Hiroaki et al. performed phase I and phase II clinical trials on SVN-2B vaccines (human leucocyte antigen-A24-restricted antigenic peptide) and interferon $\beta$ for advanced PC. A good immune reaction was demonstrated for the SVN-2B peptide vaccines with a better objective tumor response rate than placebo, indicating SVN-2B peptide vaccination could be a potential novel approach for PC and that IFN $\beta$ is a good adjuvant for peptide vaccination therapy. Thus, SVN-2B peptide vaccination with IFN $\beta$ adjuvant therapy seems to be an effective and novel strategy for future vaccination research [22].

Altogether, these studies suggest the potential of peptide cancer vaccines in treating metastatic PC depending on effective combination with other immune agents that induce a more comprehensive immune response to $\mathrm{PC}$, indicating that peptide vaccination targeting $\mathrm{PC}$ may be safe and may result in a potentially beneficial immune response with co-treatment of an immune adjuvant.

\section{Vaccine treatments targeting metastatic PC Vaccines targeting the TME}

TME plays a significant role in metastasis and progression of PC. TME comprises blood vessels and extracellular matrix (ECM), as well as various immune and other cell types. PC chemo-resistance is partly due to the dense fibrotic TME and lack of CD8+ T cells infiltrating. Moreover, cytokines secreted by cells in the TME regulate immune functions, which lead to mass immune responses and promote tumor progression as well as metastasis. Additionally, various components in the TME play both supportive and inhibitory roles in PC metastasis. For example, ECM prevents cancer cell migration but also inhibits T cell attack on PC cells. Various types of vessels transport immune cells to kill PC cells but also provide necessary nutrients to feed PC cells. Therefore, precise and effective strategies to inhibit metastasis by regulating the TME and targeting immune cells in TME are required.

Although vaccines can induce immune cells to attack PC, the TME of PC acts like a barrier that prevents a large number of immune cells from penetrating into it. The TME of PC is characterized by a fibroinflammatory and immunosuppressive stroma. The goal of vaccination is to enhance the ability of tumor-specific $\mathrm{T}$ cells and improve direct delivery of immune stimulatory agents into the TME. The key to achieve a positive outcome with vaccination requires both killing tumor cells and the appropriate utilization of adjuvants such as TLR ligands and stimulator of interferon genes (STING) agonists, which can activate DCs and helping DCs infiltrate into TME [23]. Therefore, the combination of vaccine immune therapy and novel therapies targeting the TME could be a promising strategy to improve clinical outcome in PC patients, such as enhancing $\mathrm{T}$ cell infiltration by hyaluronidase, strengthening immune cells by IL-2, IL12, as well as reducing the barrier function of TME by TGF- $\beta$ inhibitor.

Together these studies indicate that a strategy targeting the TME to establish a susceptible environment for vaccine function and tumor-specific $\mathrm{T}$ cells may be an effective strategy to inhibit PC metastasis.

\section{Vaccination and immune cells} Immune cell phenotypes and PC metastasis

There are various kinds of immune cells associated with tumor metastasis and progression such as monocytederived macrophages, tumor-associated macrophages, tumor-associated neutrophils, myeloid-derived suppressor cells (MDSCs), natural killer cell, cytotoxic lymphocyte (CTL) and tumor infiltrating lymphocytes. These cells have capacity to block CD8+ T-cell-mediated tumor killing which as a result lead to the resistance against novel therapies such as checkpoint inhibitor targeting and cancer vaccines [24]. Therefore, understanding the role and function of the immune cells of PC is significant to increase the efficacy of vaccines. Several important immune cells have two phenotypes by producing different cytokines, such as $\mathrm{T}$ cells, in which Th1 represents a pro-inflammatory phenotype, and Th2 represents an immunosuppressive phenotype. Monocyte-derived macrophages are another immune cell that has two phenotypes: the pro-inflammatory and cytotoxic M1 type and the immune-suppressive M2 type. Tumor-associated macrophages (TAMs) are associated with both the immune cells and PC cells that promote metastasis and progression of $\mathrm{PC}$ and increase chemoresistance of PC [25]. Therapies targeting TAMs in cancer, including Trabectedin [26] and Docetaxel [27], can reduce pro-tumor TAMs and inhibit metastasis.

Tumor-associated neutrophils (TAN) are another type of immune cell in the TME with two types: TAN1 (inflammatory type) and TAN2 (immunosuppressive type) [28]. Furthermore, natural killer $\mathrm{T}$ (NKT) cells include NKT1 (anti-tumor) and NKT2 (pro-tumor). Because TAN and NKT play a significant role in regulating immune response, both of TAN and NKT are probably potential targets to inhibit PC metastasis. Myeloid-derived suppressor cells (MDSCs) suppress T cells in the TME, resulting in metastasis. Therefore, inhibition of MDSCs can be a novel therapy to reduce the metastatic potential of PC. 


\section{Vaccination therapies targeting immune cells}

The TME of PC contains various immune suppressive cells that express pro-tumor cytokines. Altering the characteristics of these cells by vaccination may represent an effective immunotherapy strategy. Immune therapies can disrupt the immune-suppressive impact on T-cell function and enhance tumor antigen delivery with vaccines. Tumor antigens in PC such as Kras, telomerase, enolase, WT1, and mesothelin, among others, can stimulate cancer-specific T-cell responses. For example, CD4+ and CD8+ T cells that specifically target mesothelin can be enhanced by vaccination [29]. Vaccines such as live attenuated bacteria expressing mesothelin (CRS-207) can stimulate tumor-specific $T$ cells while inhibiting the function of pro-tumor T cells. GM-CSF transduced allogeneic whole tumor cell vaccine (GVAX) can improve the immune system in PC by stimulating anti-tumor $\mathrm{T}$ cell infiltration and myeloid cell activation [30, 31] (Fig. 2).
Together these studies show that vaccination can alter the characteristics of the immune system in TME. Therefore, combination of inhibitors of tumor-suppressing cells with vaccination therapies and increasing protective phenotypes of those immune cells may be a promising strategy.

\section{Vaccines and immune checkpoints \\ PD-L1/PD-1 and vaccines}

Programmed death receptor ligand 1 (PD-L1) is overexpressed in many types of malignancies and is a crucial factor in helping tumor cells achieve immune escape. PD-L1 and PD-L2 are ligands for PD-1 that activate immune checkpoints. PD-L1 inhibits the antitumor effects of DC immunization and regulates Tregs and IFN- $\gamma$ (upregulating IL-10 and IL-2 as well as downregulating IFN- $\gamma$ ), and its expression is statistically correlated with poor outcome in PC patients [32]. ARF6

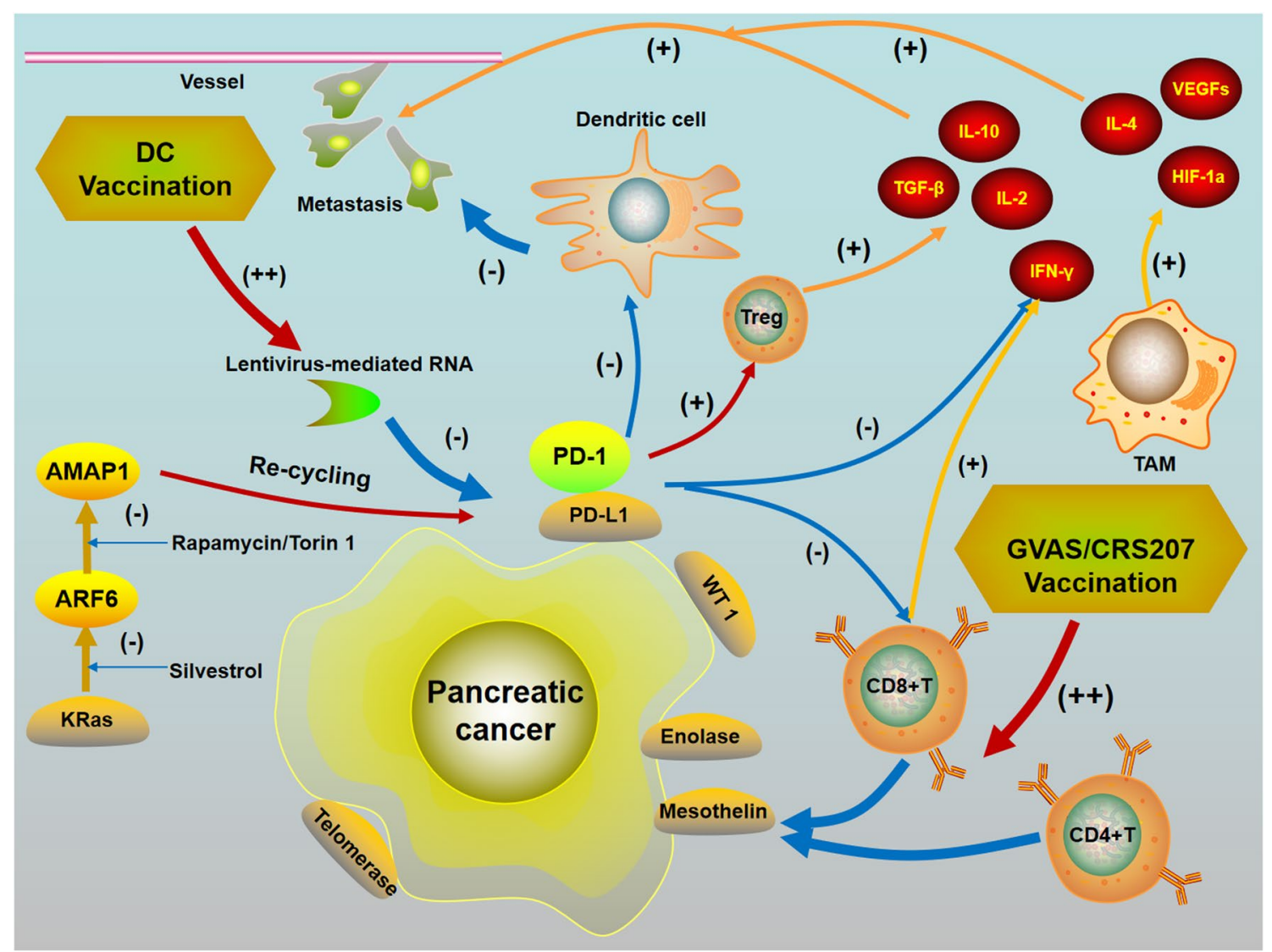

Fig. 2 Vaccinations can inhibit the release of various pro-tumor cytokines. Programmed death-1 ligand (PD-L1) inhibits the antitumor effects of Dendritic cell (DC) immunization and regulates regulatory T cells (Treg) which as a result upregulate IL-10 and IL-2 as well as downregulate IFN- $\gamma$. 2) ARF6/AMAP1 signaling pathway is closely correlated with the intracellular recycling of PD-L1. Inhibition of ARF6/AMAP1 can reduce PD-L1 cell surface expression. 3) Tumor antigens in PC such as telomerase, enolase, WT1, and mesothelin can stimulate cancer-specific T-cell responses. For example, CD4+ and CD8+ T cells that specifically target mesothelin can be enhanced by vaccination. 4) Vaccines such as live attenuated bacteria expressing mesothelin (CRS-207) and GM-CSF transduced allogeneic whole tumor cell vaccine (GVAX) can improve the immune system in PC by stimulating anti-tumor T cell infiltration. (+) Promote $(-)$ Inhibit 
and AMAP1 are overexpressed in PC and ARF6 activation can down-regulate E-cadherin and upregulate focal adhesion turnover, thus promoting tumor invasion and metastasis [33-35]. KRAS and TP53 oncogenic mutations help activate the ARF6/AMAP1 signaling pathway and both ARF6/AMAP1 and KRAS/TP53 oncogenic mutations were closely correlated with the intracellular recycling of PD-L1. KRAS and TP53 oncoproteins enhance PD-L1 expression via ARF6 and AMAP1, resulting in PC progression and metastasis [36]. Therefore, inhibition of ARF6/AMAP1 or KRAS/TP53 can reduce PD-L1 cell surface expression [36]. The eIF4A inhibitor silvestrol can block ARF6 expression and mTOR inhibitors rapamycin and Torin 1 can inhibit AMAP1. These inhibitors may be novel drugs that can target metastatic PC as immune checkpoint blockades (ICBs) [37, 38].

In PC, blocking PD-1/PD-L1 alone has no significantly positive impact due to the lack of natural infiltration of immune cells such as T cells [39]. Although the potential therapeutic effects of ICB have been demonstrated, most PC patients treated with anti-PD-L1/PD-1 mono-therapy do not obtain satisfying outcomes, with frequent tumor regression or metastasis. Hence, cancer vaccines with ICBs may improve the clinical outcomes of patients with $\mathrm{PC}$ by activating $\mathrm{T}$ cells and inducing effective anti-tumor immunity because anti PD-1 can increase effector CD8+ $\mathrm{T}$ cells and secretion of IFN- $\gamma$ in the TME, while vaccination could block the immunosuppressive pathways, inhibit immune-inhibiting $\mathrm{T}$ cells and reduce CTLA-4 expression on $\mathrm{T}$ cells. Bassani-Sternberg et al. hypothesized that vaccination can improve tumor immune recognition in metastatic $\mathrm{PC}$, thus increasing the response to PD-1/PD-L1 blockade [11]. Recently, increasing researches have explored combining inhibitors of PD-1/ PD-L1 and cancer vaccines for metastatic PC. Preclinical data indicated that $\mathrm{DC}$ vaccines combined with ICBs could generate a synergistic effect with low additional toxicity [40]. Moreover, PD-L1 knockdown by lentivirus-mediated RNA interference combined with DC vaccination may enhance $\mathrm{T}$ cell activity in $\mathrm{PC}$ on inhibiting metastasis and improving survival [41]. Furthermore, the cancer vaccine GVAX combined with anti PD-1/PD-L1 therapy increased CD8 $+\mathrm{T}$ infiltration into pancreatic tumors and improved patient survival [42]. Soares et al. explored the combination of vaccine with PD-1 antibody in an animal study and found that this novel combination can bring much more benefit and improve survival compared with anti-PD-1 alone.

Additionally, gastrin, a gastrointestinal peptide, not only promotes the development of PC but also induce EMT and metastasis of PC by increasing $\beta$-catenin $[43,44]$. Osborne et al. recently demonstrated that the tumor vaccine polyclonal antibody stimulator (PAS), which selectively targets gastrin, induces system to produce specific polyclonal anti-gastrin antibodies and then decrease PC growth and metastases by downregulating dense desmoplastic fibrosis in the TME, inducing $\mathrm{T}$ cell activation, and altering the TME to make it more responsive to immunotherapy with a PD-1 immune checkpoint antibody [45]. We suggest that PAS co-treated with antiPD-1 immunotherapy may be a novel strategy to inhibit PC metastasis.

Therefore, combining PD-1 or PD-L1 antibody therapy with a vaccine that induces the effects of $\mathrm{T}$ cell could represent a novel and efficient strategy for metastatic PC treatment [42]. Thus, a combination of cancer vaccine with these drugs could enhance induction of the immune response and vaccination is thus a potential method to enhance the ability of effector $\mathrm{T}$ cells to enter the TME and may play a role in enhancing anti-PD-1 therapy.

\section{CTLA-4 and vaccines}

Another well-known immune checkpoint is cytotoxic $\mathrm{T}$ lymphocyte-associated antigen-4 (CTLA-4), which plays a significant role in tumor tolerance [46]. Antibodies targeting CTLA-4 can block the combination of CTLA-4 with its ligands B7 on APCs, which as a result, can inhibit the apoptosis of activated lymphocytes, thereby upregulating the immune response and producing antitumor activity [47]. Ipilimumab was the first ICB targeting CTLA-4 to be approved by FDA for cancer treatment.

CTLA-4 is mainly expressed on Tregs, which decrease $\mathrm{T}$ cell immune responses and help tumor cells realize immune escape. Tregs can express CD25 (the IL-2 receptor), which is vital for their immunosuppressive function [48]. In addition, CTLA-4 inhibits the T cell response by competing for B7 ligands CD80 and CD86. CD80 and CD86 which are expressed on APCs, provide co-stimulatory signals to T cells via CD28. Notably, CD80 and CD86 have a closer affinity to CTLA-4 than the co-receptor CD28. Hence, inhibition of CTLA-4 interaction with CD80 and CD86 can induce CD4 T cell infiltration into tumors. Bengsch et al. showed that antibodies targeting CTLA-4 can stimulate CD4+ T cell infiltration into tumors but cannot enhance $\mathrm{CD} 8+\mathrm{T}$ cell infiltration into PC tumors. Therefore, other methods such as vaccine therapies that can improve CD8+ T cell ability are likely necessary to strengthen the killing capacity of CTLA4-targeted antibodies in PC [49]. Thus, cancer vaccines that can stimulate CD8+ T cell activation may be effective in targeting $\mathrm{PC}$ metastasis.

Zaidi et al. combined anti-CTLA-4 approaches with an antigen-specific DC vaccine in murine PC models and found that this combination significantly elevated vaccine-induced $C D 4+T$ cells as well as $C D 8+T$ cells in $\mathrm{PC}$, providing a novel idea of the combination of vaccine 
therapy with ICB to drive T cell infiltration in murine PC models [50]. Hence, the combination of ICB, immuneenhancers and vaccination represents a potent therapeutic strategy to target PC metastasis.

In conclusion, recent developments in chemotherapy have improved the overall survival in PC patients, but the mortality rates remain high. The use of multiple vaccines has become more prominent due to demonstrated immunogenic and safe outcomes, but the application of vaccination alone can induce immunosuppressive Tregs and MDSCs. Above all, ICBs such as anti-PD-1 and antiCTLA- 4 have improved outcome in PC patients. The combination of cancer vaccines with checkpoint blockade (anti-CTLA-4, anti-PD-1) might strengthen the antitumor effects with stronger immune response but lower toxicity.

\section{Boosting vaccines with adjuvants}

To overcome the immunosuppressive characteristic of $\mathrm{PC}$ and lack of enough specific antigens, adjuvants are needed for PC immunotherapy. Current literature has demonstrated the potency of cancer vaccines in combination with adjuvants to obtain efficient immune responses and delivery in metastatic PC. Adjuvants have little ability to eliminate $\mathrm{PC}$ cells but can promote the immunogenicity of vaccination.

GM-CSF is an important cytokine that can promote the antigen-presenting ability of DCs. GVAX is a whole cell-based vaccine that can express GM-CSF. As an adjuvant, Cyclophosphamide(Cy) can inhibit the immunesuppressive Tregs, and GVAX vaccination combined with Cy improved the overall survival rate of metastatic PC patients compared with GVAX vaccination alone(130 vs. 69 days) [51].

Additionally, GVAX combined with Listeria monocytogenes (Lm)-based vaccine has shown positive outcomes of inducing stronger DC function and activating elevated cellular immunity. CRS-207 is a live-attenuated strain of Lm engineered to express mesothelin, a TAA of PC. Le et al. demonstrated that CRS-207 boosted the clinical outcomes of Cy/GVAX treatment and Cy/GVAX followed by CRS-207 significantly improved overall survival compared with Cy/GVAX alone in metastatic PC patients [29]. Based on the observed survival and favorable safety profile, Cy/GVAX and CRS-207 are being explored as a treatment for PC.

Besides, CD40, a surface member of the TNF receptor superfamily, can strengthen vaccine effects and antigenspecific CD8+ T cells, dependent on the increased IL-12 (critical $\mathrm{T}$ cell stimulatory cytokines) secreted by CD40expressing DCs [52].

Furthermore, Blair et al. showed that indoleamine2,3-dioxygenase (IDO1) which is expressed on PC, can induce the resistance to immunotherapy. GVAX vaccine can induce IDO1 expression but IDO1 overexpression is closely associated with poor survival following GVAX treatment. Thus an IDO1 inhibitor (EOS200271) enhanced antitumor efficacy of GVAX by increasing T cell amount and function in PC. These results indicate that the combination of vaccine and IDO1 inhibitor could be a novel and effective therapeutic strategy for PC metastasis and suggest the importance of inhibiting IDO1 in vaccine therapy [53].

To make the best use of adjuvant on vaccines, nanoparticles or microparticles have been found as carriers for co-delivering vaccine adjuvant and antigen and protecting vaccine components from hydrolyzing, which can enhance immunogenicity while maintaining their original efficacy [54]. Particles containing both antigen and adjuvant showed better outcomes than antigen only. Particles combine particulate cancer vaccines and other immunomodulation reagents will become a novel strategy in PC immunotherapy.

In conclusion, vaccines targeting tumors have shown great promise in the safety and feasibility in metastatic PC treatment. To strongly enhance immune response, cancer adjuvants should be co-administered with vaccines to achieve more effective delivery in TME, as adjuvants can enhance the immunogenicity of the vaccine. We suggest that the combination immuno-therapies and vaccines are effective approaches in cancer immunotherapy to decrease immunosuppression and augment anticancer effects.

\section{Conclusion}

In this review, numerous underlying mechanisms of vaccines and novel therapies for metastatic PC have been discussed. Immune system response to TME play a dominant role in affecting the metastatic capacity of PC cells. Vaccines that induce a T-cell response may enhance the efficacy of immunotherapy. Therefore, the combination of vaccine therapies with drugs targeting the TME to enhance the infiltration of $\mathrm{T}$ cells can strengthen the effect of inhibiting PC metastasis. Besides, we highlight the importance of inhibiting immune checkpoints in the application of vaccine therapy. This approach may contribute to further optimization of the best combination of drugs and vaccines for metastatic PC patients.

\footnotetext{
Acknowledgements

We acknowledge all the participants in searching, analyzing and concluding those studies that contributed to this piece of work and all the collaborators who make such studies possible. We acknowledge the clinic staff and managers of Peking Union Medical College Hospital, Chinese Academy of Medical Sciences and Peking Union Medical College for their valuable contributions to this research.
} 


\section{Authors' contributions}

Study design: WHL and WTL; Literature search: WHL and WTL; Study selection: WHL; Study draft and revision: WHL and WTL; Suggestions: ZC, GY, YZL, JDQ, GYC, LY, FYZ, LFZ. Article guarantor: TPZ. All authors read and approved the final manuscript.

\section{Funding}

No funding declared.

\section{Data sharing}

No additional data available.

\section{Ethics approval and consent to participate}

Not required.

\section{Consent for publication}

We exceedingly hope that this manuscript could be accepted and published by Cancer Cell International.

\section{Competing interests}

The authors declare that they have no competing interests.

\section{Author details}

1 Department of General Surgery, Peking Union Medical College Hospital, Chinese Academy of Medical Sciences and Peking Union Medical College, No. 1 Shuaifuyuan, Wangfujing Street, Beijing 100730, China. ${ }^{2}$ Department of Mechanical Engineering, Tsinghua University, Beijing 100084, China.

${ }^{3}$ Department of Nuclear Medicine, Peking Union Medical College Hospital, Chinese Academy of Medical Sciences and Peking Union Medical College, Beijing 100730, China. ${ }^{4}$ Clinical Immunology Center, Chinese Academy of Medical Sciences and Peking Union Medical College, Beijing 100730, China.

\section{Received: 3 October 2019 Accepted: 20 February 2020}

Published online: 04 March 2020

\section{References}

1. Makohon-Moore A, lacobuzio-Donahue CA. Pancreatic cancer biology and genetics from an evolutionary perspective. Nat Rev Cancer. 2016;16(9):553-65.

2. Miyazawa M, et al. Phase II clinical trial using novel peptide cocktail vaccine as a postoperative adjuvant treatment for surgically resected pancreatic cancer patients. Int J Cancer. 2017;140(4):973-82.

3. Scott AM, Wolchok JD, Old LJ. Antibody therapy of cancer. Nat Rev Cancer. 2012;12(4):278-87.

4. Wurz GT, Kao CJ, DeGregorio MW. Novel cancer antigens for personalized immunotherapies: latest evidence and clinical potential. Ther Adv Med Oncol. 2016;8(1):4-31

5. Dhillon S. Dinutuximab: first global approval. Drugs. 2015;75(8):923-7.

6. Fukuda M. Possible roles of tumor-associated carbohydrate antigens. Cancer Res. 1996;56(10):2237-44.

7. Ugorski M, Laskowska A. Sialyl Lewis(a): a tumor-associated carbohydrate antigen involved in adhesion and metastatic potential of cancer cells. Acta Biochim Pol. 2002:49(2):303-11.

8. Weitzenfeld P, Bournazos S, Ravetch JV. Antibodies targeting sialyl Lewis A mediate tumor clearance through distinct effector pathways. J Clin Invest. 2019;129:3952-62

9. Ward JP, Gubin MM, Schreiber RD. The role of neoantigens in naturally occurring and therapeutically induced immune responses to cancer. Adv Immunol. 2016;130:25-74

10. Zhang $X$, et al. Personalized cancer vaccines: targeting the cancer mutanome. Vaccine. 2017;35(7):1094-100.

11. Bassani-Sternberg $M$, et al. A phase lb study of the combination of personalized autologous dendritic cell vaccine, aspirin, and standard of care adjuvant chemotherapy followed by nivolumab for resected pancreatic adenocarcinoma-a proof of antigen discovery feasibility in three patients. Front Immunol. 2019:10:1832.

12. Cao J, et al. DNA vaccines targeting the encoded antigens to dendritic cells induce potent antitumor immunity in mice. BMC Immunol. 2013;14:39.
13. Palucka K, et al. Dendritic cells and immunity against cancer. J Intern Med. 2011;269(1):64-73.

14. Maccalli C, Parmiani G, Ferrone S. Immunomodulating and immunoresistance properties of cancer-initiating cells: implications for the clinical success of immunotherapy. Immunol Invest. 2017;46(3):221-38.

15. Pan $L$, et al. Magnetic resonance imaging monitoring therapeutic response to dendritic cell vaccine in murine orthotopic pancreatic cancer models. Am J Cancer Res. 2019;9(3):562-73.

16. Rong Y, et al. A phase I pilot trial of MUC1-peptide-pulsed dendritic cells in the treatment of advanced pancreatic cancer. Clin Exp Med. 2012;12(3):173-80.

17. Mehrotra $\mathrm{S}$, et al. Vaccination with poly $(\mathrm{IC}: \mathrm{LC})$ and peptide-pulsed autologous dendritic cells in patients with pancreatic cancer. J Hematol Oncol. 2017:10(1):82.

18. Townsend AR, et al. The epitopes of influenza nucleoprotein recognized by cytotoxic T lymphocytes can be defined with short synthetic peptides. Cell. 1986;44(6):959-68.

19. Gjertsen MK, et al. Ex vivo ras peptide vaccination in patients with advanced pancreatic cancer: results of a phase I/II study. Int J Cancer. 1996:65(4):450-3.

20. Overwijk WW, Theoret MR, Restifo NP. The future of interleukin-2: enhancing therapeutic anticancer vaccines. Cancer J Sci Am. 2000;6(Suppl 1):S76-80.

21. Gjertsen MK, et al. Intradermal ras peptide vaccination with granulocytemacrophage colony-stimulating factor as adjuvant: clinical and immunological responses in patients with pancreatic adenocarcinoma. Int J Cancer. 2001;92(3):441-50.

22. Shima $\mathrm{H}$, et al. Randomized phase II trial of survivin $2 \mathrm{~B}$ peptide vaccination for patients with HLA-A24-positive pancreatic adenocarcinoma. Cancer Sci. 2019;110(8):2378-85.

23. Chen Q, Sun L, Chen ZJ. Regulation and function of the CGAS-STING pathway of cytosolic DNA sensing. Nat Immunol. 2016;17(10):1142-9.

24. Martinez-Bosch N, Vinaixa J, Navarro P. Immune evasion in pancreatic cancer: from mechanisms to therapy. Cancers (Basel). 2018. https://doi. org/10.3390/cancers10010006.

25. Ostuni R, et al. Macrophages and cancer: from mechanisms to therapeutic implications. Trends Immunol. 2015;36(4):229-39.

26. Germano $\mathrm{G}$, et al. Role of macrophage targeting in the antitumor activity of trabectedin. Cancer Cell. 2013;23(2):249-62.

27. Kodumudi $\mathrm{KN}$, et al. A novel chemoimmunomodulating property of docetaxel: suppression of myeloid-derived suppressor cells in tumor bearers. Clin Cancer Res. 2010;16(18):4583-94.

28. Fridlender ZG, Albelda SM. Tumor-associated neutrophils: friend or foe? Carcinogenesis. 2012;33(5):949-55.

29. Le DT, et al. Safety and survival with GVAX pancreas prime and Listeria Monocytogenes-expressing mesothelin (CRS-207) boost vaccines for metastatic pancreatic cancer. J Clin Oncol. 2015;33(12):1325-33.

30. Tsujikawa T, et al. Quantitative multiplex immunohistochemistry reveals myeloid-inflamed tumor-immune complexity associated with poor prognosis. Cell Rep. 2017;19(1):203-17.

31. Le DT, et al. Results from a Phase IIb, randomized, multicenter study of GVAX pancreas and CRS-207 compared with chemotherapy in adults with previously treated metastatic pancreatic adenocarcinoma (ECLIPSE Study). Clin Cancer Res. 2019;25:5493-502.

32. Nomi $T$, et al. Clinical significance and therapeutic potential of the programmed death-1 ligand/programmed death-1 pathway in human pancreatic cancer. Clin Cancer Res. 2007;13(7):2151-7.

33. Logsdon CD, Ji B. Ras activity in acinar cells links chronic pancreatitis and pancreatic cancer. Clin Gastroenterol Hepatol. 2009;7(11 Suppl):S40-3.

34. Guerra $C$, et al. Pancreatitis-induced inflammation contributes to pancreatic cancer by inhibiting oncogene-induced senescence. Cancer Cell. 2011:19(6):728-39.

35. Rhim AD, Stanger BZ. Molecular biology of pancreatic ductal adenocarcinoma progression: aberrant activation of developmental pathways. Prog Mol Biol Transl Sci. 2010;97:41-78.

36. Hashimoto $S$, et al. ARF6 and AMAP1 are major targets of KRAS and TP53 mutations to promote invasion, PD-L1 dynamics, and immune evasion of pancreatic cancer. Proc Natl Acad Sci USA. 2019:116:17450-9.

37. Bordeleau ME, et al. Therapeutic suppression of translation initiation modulates chemosensitivity in a mouse lymphoma model. J Clin Invest. 2008;118(7):2651-60. 
38. Thoreen CC, et al. A unifying model for mTORC1-mediated regulation of mRNA translation. Nature. 2012:485(7396):109-13.

39. Ino $Y$, et al. Immune cell infiltration as an indicator of the immune microenvironment of pancreatic cancer. Br J Cancer. 2013;108(4):914-23.

40. Fu J, et al. Preclinical evidence that PD1 blockade cooperates with cancer vaccine TEGVAX to elicit regression of established tumors. Cancer Res. 2014;74(15):4042-52.

41. Wang J, et al. Lentivirus-mediated RNA interference targeting programmed death receptor ligand 1 increases the immunologic anti-tumor effect of dendritic cell vaccination against pancreatic cancer in SCID-hu mice. Oncol Lett. 2019;18(2):1539-47.

42. Soares KC, et al. PD-1/PD-L1 blockade together with vaccine therapy facilitates effector T-cell infiltration into pancreatic tumors. J Immunother 2015;38(1):1-11.

43. Smith JP, et al. Gastrin regulates growth of human pancreatic cancer in a tonic and autocrine fashion. Am J Physiol. 1996;270(5 Pt 2):R1078-84.

44. Zhuang K, et al. Gastrin promotes the metastasis of gastric carcinoma through the beta-catenin/TCF-4 pathway. Oncol Rep. 2016;36(3):1369-76.

45. Osborne N, et al. Vaccine against gastrin, polyclonal antibody stimulator, decreases pancreatic cancer metastases. Am J Physiol Gastrointest Liver Physiol. 2019;317:G682-93.

46. Popovic A, Jaffee EM, Zaidi N. Emerging strategies for combination checkpoint modulators in cancer immunotherapy. J Clin Invest. 2018;128(8):3209-18.

47. Quezada SA, et al. CTLA4 blockade and GM-CSF combination immunotherapy alters the intratumor balance of effector and regulatory $T$ cells. J Clin Invest. 2006;116(7):1935-45.
48. Rech AJ, et al. CD25 blockade depletes and selectively reprograms regulatory $T$ cells in concert with immunotherapy in cancer patients. Sci Trans Med. 2012:4(134):134ra62

49. Bengsch F, et al. CTLA-4/CD80 pathway regulates $T$ cell infiltration into pancreatic cancer. Cancer Immunol Immunother. 2017:66(12):1609-17.

50. Zaidi N, et al. Anti-CTLA-4 synergizes with dendritic cell-targeted vaccine to promote IL-3-dependent CD4(+) effector T cell infiltration into murine pancreatic tumors. Ann NY Acad Sci. 2019;1445(1):62-73.

51. Laheru $D$, et al. Allogeneic granulocyte macrophage colony-stimulating factor-secreting tumor immunotherapy alone or in sequence with cyclophosphamide for metastatic pancreatic cancer: a pilot study of safety, feasibility, and immune activation. Clin Cancer Res. 2008;14(5):1455-63.

52. Vonderheide RH, Glennie MJ. Agonistic CD40 antibodies and cancer therapy. Clin Cancer Res. 2013;19(5):1035-43.

53. Blair $A B$, et al. IDO1 inhibition potentiates vaccine-induced immunity against pancreatic adenocarcinoma. J Clin Invest. 2019;129(4):1742-55.

54. Saung MT, et al. Particulate carrier systems as adjuvants for cancer vaccines. Biomater Sci. 2019;7:4873.

\section{Publisher's Note}

Springer Nature remains neutral with regard to jurisdictional claims in published maps and institutional affiliations.
Ready to submit your research? Choose BMC and benefit from:

- fast, convenient online submission

- thorough peer review by experienced researchers in your field

- rapid publication on acceptance

- support for research data, including large and complex data types

- gold Open Access which fosters wider collaboration and increased citations

- maximum visibility for your research: over $100 \mathrm{M}$ website views per year

At BMC, research is always in progress.

Learn more biomedcentral.com/submissions 